\title{
Amostragem probabilística estratificada por pontos para estimar a área cultivada com soja
}

\author{
Marcos Adami ${ }^{(1)}$, Rodrigo Rizzi(2), Maurício Alves Moreira( ${ }^{(1)}$, \\ Bernardo Friedrich Theodor Rudorff(1) e Camila Cossetin Ferreira ${ }^{(1)}$
}

(1)Instituto Nacional de Pesquisas Espaciais, Avenida dos Astronautas, no 1758, CEP 12227-010 São José dos Campos, SP.
E-mail: adami@dsr.inpe.br, moreira@dsr.inpe.br, bernardo@dsr.inpe.br, camila.ferreira@cptec.inpe.br (2)Universidade Federal de Pelotas,
Campus Universitário, CEP 96001-970 Capão do Leão, RS. E-mail: rodrigo.rizzi@ufpel.edu.br

Resumo - O objetivo deste trabalho foi avaliar o desempenho de um modelo probabilístico de amostragem estratificada por pontos, e definir um tamanho de amostra adequado para estimar a área cultivada com soja no Rio Grande do Sul. A área foi estratificada de acordo com a percentagem de soja cultivada em cada município do estado: menor que 20, de 20 a 40 e maior que $40 \%$. Foram avaliadas estimativas obtidas por meio de seis tamanhos de amostras, resultantes da combinação de três níveis de significância $(10,5$ e 1\%) e dois valores de erro amostral (5 e 2,5\%). Para cada tamanho de amostra, foram realizados 400 sorteios aleatórios. As estimativas foram avaliadas com base na área de soja obtida de um mapa temático de referência proveniente de uma cuidadosa classificação automática e visual de imagens multitemporais dos satélites TM/Landsat-5 e ETM+/Landsat-7 disponível para a safra 2000/2001. A área de soja no Rio Grande do Sul pode ser estimada por meio de um modelo de amostragem probabilística estratificada por pontos, sendo que a melhor estimativa é obtida para o maior tamanho amostral (1.990 pontos), com diferença de apenas $-0,14 \%$ em relação à estimativa do mapa de referência e um coeficiente de variação de 6,98\%.

Termos para indexação: Glycine max, estatísticas agrícolas, imagem de satélite, imagens multitemporais, modelagem, sistemas de informação geográfica.

\section{Probabilistic stratified point sampling to estimate soybean crop area}

\begin{abstract}
The objective of this work was to evaluate the performance of a probabilistic sampling model stratified by points and to define an appropriate sample size to estimate the cultivated soybean area in the state of Rio Grande do Sul, Brazil. The area was stratified according to the percentage of soybean cultivated in each state municipality: less than 20, from 20 to 40 and more than $40 \%$. Estimates were evaluated based on six sample sizes, resulting from the combination of three significance levels $(10,5$ and $1 \%)$ and two sampling errors (5 and 2,5\%), choosing 400 random samples for each sample size. The estimates were compared to a reference soybean thematic map available for the crop year 2000/2001 that was derived from a careful automatic and visual classification of multitemporal TM/Landsat-5 and ETM+/Landsat-7 images. The soybean area in Rio Grande do Sul State can be estimated through a probabilistic sampling model stratified by points with best estimates obtained for the largest sample size (1,990 points), which differed $-0.14 \%$ in relation to the estimate of the reference map and had a coefficient of variation of $6.98 \%$.
\end{abstract}

Index terms: Glycine max, agricultural statistics, satellite image, multitemporal images, modeling, geographic information systems.

\section{Introdução}

A confiabilidade e a oportunidade são dois importantes critérios que devem ser considerados na estimativa de safras agrícolas. A confiabilidade está relacionada à precisão da estimativa, enquanto a oportunidade refere-se ao momento em que esta é divulgada. $\mathrm{O}$ estabelecimento de um método para estimar a produção das principais culturas agrícolas que atenda a tais critérios é de grande importância para os integrantes do agronegócio e para o governo.

No Brasil, o Instituto Brasileiro de Geografia e Estatística (IBGE) e a Companhia Nacional de Abastecimento (Conab) são os órgãos oficiais responsáveis pela estimativa de safras. As informações geradas por esses órgãos englobam área plantada, 
produtividade e produção das culturas. Tais estimativas são realizadas predominantemente de forma subjetiva, uma vez que as informações levantadas têm por base a opinião de agentes ligados ao setor. Em função dessa subjetividade, não é possível fazer uma análise quantitativa dos erros e das incertezas envolvidos, o que pode dar margem a falsas interpretações e especulações (Pino, 2001). Mesmo não sendo possível aferir os erros, a metodologia utilizada por órgãos oficiais é muito empregada por ser de baixo custo e de fácil aplicação.

Vários trabalhos de levantamento da área plantada de grandes culturas agrícolas sugerem o uso de imagens de satélites de sensoriamento remoto para obter estimativas mais objetivas, de modo a reduzir a subjetividade da metodologia utilizada pelos órgãos oficiais (Rizzi \& Rudorff, 2005; Moreira et al., 2007; Rudorff et al., 2010). Contudo, a despeito da crescente disponibilidade de imagens oriundas de novos sensores com características apropriadas a esse fim, a grande extensão territorial brasileira, a elevada dinâmica espaço-temporal da atividade agrícola e, principalmente, a dificuldade de obtenção de imagens livres de nuvens (Sano et al., 2007; Sugawara et al., 2008) restringem o potencial uso operacional dessas imagens a algumas regiões e a culturas específicas (Rudorff et al., 2005).

Por essa razão, esforços foram empregados a fim de estimar a área das principais culturas agrícolas do país, por meio de modelo probabilístico de amostragem (Mueller et al., 1988; Epiphanio et al., 2002; Luiz et al., 2002, 2006; Luiz, 2003; Adami et al., 2004, 2005, 2007; Rizzi et al., 2006). Os trabalhos de Epiphanio et al. (2002), Luiz et al. (2002) e Luiz (2003) propõem o uso de imagens de satélite como grade amostral e consideram o pixel como a menor área amostrada. Para aplicar esse modelo de amostragem por ponto, em escala estadual, Luiz et al. (2006) utilizaram amostragem aleatória estratificada, cuja fonte de dados para estratificação são as estimativas municipais geradas pelo IBGE. Uma vez estabelecidos os estratos, são sorteados os municípios e, de cada um deles, é extraída uma amostra (pixels) a ser vistoriada in loco. Essa abordagem foi empregada para estimar a área cultivada com soja nos Estados do Rio Grande de Sul, Paraná e Mato Grosso, no âmbito do projeto Geosafras (Figueiredo, 2005).

Os modelos de amostragem probabilísticos podem fornecer estimativas de área cultivada, independentemente da disponibilidade de imagens, visto que somente alguns locais necessitam ser vistoriados in loco. Além disso, eles fornecem o erro associado à estimativa e possibilitam a inferência estatística sobre os resultados. Contudo, o principal obstáculo à utilização operacional desses modelos é o elevado custo para obtenção das informações acerca das áreas amostradas. Como a exatidão da estimativa está associada ao tamanho amostral, e este, por sua vez, ao custo, é fundamental que se encontre um ponto de equilíbrio entre a qualidade da estimativa e o número de amostras a ser levantado no campo.

O objetivo deste trabalho foi avaliar um modelo probabilístico de amostragem aleatória estratificado por pontos e definir o tamanho de amostra adequado para estimar a área de soja, tendo como área de estudo o Estado do Rio Grande do Sul.

\section{Material e Métodos}

A área escolhida como estudo de caso abrange 322 municípios (111.628 $\mathrm{km}^{2}$ ) do Estado do Rio Grande do Sul (Figura 1), situados entre os paralelos $27^{\circ} 03^{\prime} \mathrm{S}$ e $30^{\circ} 13^{\prime} \mathrm{S}$ e os meridianos $50^{\circ} 40^{\prime} \mathrm{W}$ e $56^{\circ} 20^{\prime} \mathrm{W}$, o que correspondeu, aproximadamente, a 90\% da área plantada com soja no Estado, na safra 2000/2001 (Instituto Brasileiro de Geografia e Estatística, 2001).

Foi utilizado um mapa temático de referência contendo a distribuição espacial das áreas de soja,

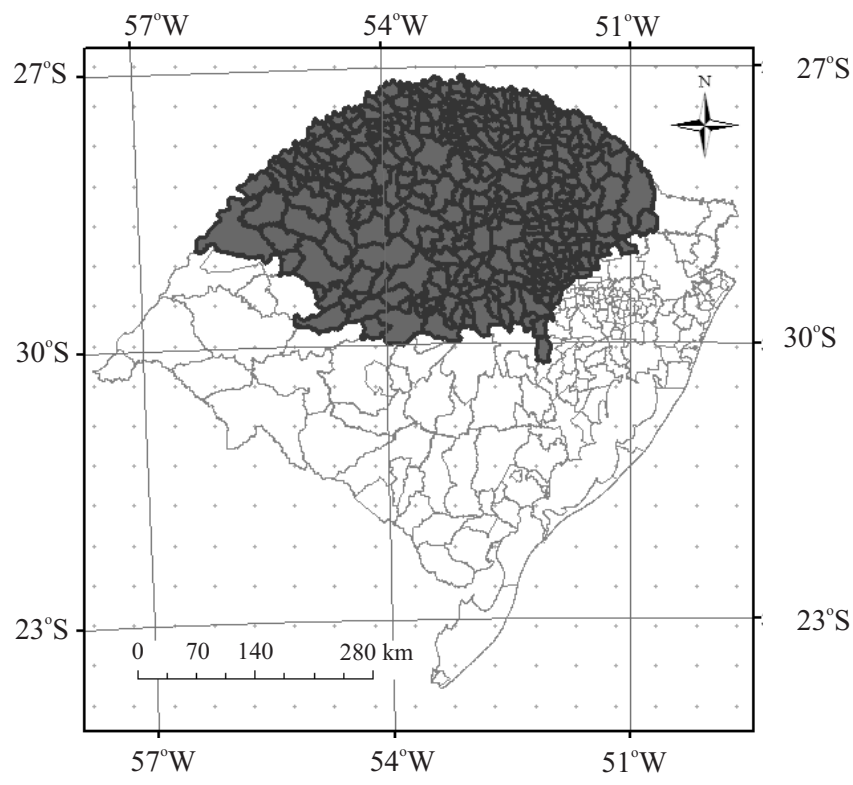

Figura 1. Localização geodésica da área de estudo. 
produzido a partir de uma criteriosa classificação automática e visual de imagens multitemporais dos satélites TM/Landsat-5 e ETM+Landsat-7, disponíveis para a safra 2000/2001 (Rizzi \& Rudorff, 2005).

Como a área de estudo contempla tanto municípios de intenso cultivo de soja como regiões onde a cultura é pouco expressiva, optou-se por um modelo de amostragem probabilística aleatória estratificada por pontos. A estratificação visa diminuir a variabilidade em populações heterogêneas e, com isso, reduzir o tamanho amostral (n) sem comprometer significativamente a exatidão da estimativa. Contudo, tal processo somente é proveitoso quando resulta em estratos homogêneos e, para tanto, deve-se tomar por base a própria variável a ser estudada ou outra altamente correlacionada (Tsiligirides, 1998). Nesse caso, a utilização de um mapa temático de referência contendo a distribuição espacial da cultura, passível de ser atualizado ao longo do tempo, parece bem adequado para a estratificação.

Assim, a partir do mapa temático de referência e dos limites políticos municipais, os estratos 1,2 e 3 foram gerados, respectivamente, com base nos seguintes limites: menor que $20 \%$, entre 20 e $40 \%$ e maior que $40 \%$ da área cultivada com soja nos municípios (Tabela 1).

Para estimar o tamanho da amostra, empregou-se a equação (Thompson, 2002):

$$
\mathrm{n}=\left[\left(\mathrm{Z}_{\alpha / 2}\right)^{2} \mathrm{pq}\right] / \mathrm{E}^{2},
$$

em que: $\mathrm{n}$ é o número total de elementos amostrais (pontos), selecionados para estimar a área com determinado nível de significância $(\alpha)$, e determinado erro de amostragem (E); p é a fração da área do estrato ocupada com soja (Tabela 1); q é fração da área do estrato não ocupada com soja (1-p) e Z é o valor crítico tabelado da função normal padrão, para $\alpha / 2$. Para relacionar o tamanho amostral à

Tabela 1. Número do estrato, número de municípios, área do estrato e área de soja obtida a partir do mapa temático de referência.

\begin{tabular}{lccc}
\hline Estrato & № de municípios & $\begin{array}{c}\text { Área do estrato } \\
\left(\mathrm{km}^{2}\right)\end{array}$ & $\begin{array}{c}\text { Área de soja } \\
\left(\mathrm{km}^{2}\right)\end{array}$ \\
\hline 1 & 176 & 60.917 & $3.752(6,2)^{(1)}$ \\
2 & 75 & 22.542 & $6.952(30,8)$ \\
3 & 71 & 28.169 & $14.225(50,5)$ \\
\hline Todos & 322 & 111.628 & $24.929(22,3)$ \\
\hline
\end{tabular}

${ }^{(1)}$ Valores entre parênteses correspondem à porcentagem da área de soja. Fonte: Rizzi \& Rudorff (2005). exatidão da estimativa, optou-se por avaliar três valores de $\alpha(10,5$ e $1 \%)$ e dois valores de $E(E=5$ e 2,5\%), o que resultou em seis tamanhos amostrais. $\mathrm{O}$ número de elementos (unidades amostrais) por estrato (nh) foi obtido pelo método de alocação de Neyman, conforme a equação (Thompson, 2002):

$$
\mathrm{nh}=\left[(\mathrm{Nh} \times \mathrm{Sh}) /\left(\sum_{\mathrm{i}=1}^{\mathrm{m}} \mathrm{Nh} \times \mathrm{Sh}\right)\right] \mathrm{n} ;
$$

em que: Nh é o número total de elementos do estrato; Sh é o desvio-padrão do estrato; n é o número total de elementos amostrais estimados pela equação anterior e i é o índice que representa o estrato de uso do solo com $\mathrm{i}=1, \ldots, \mathrm{m}$, sendo $\mathrm{m}$ o número total de estratos.

Uma vez definidos os conjuntos amostrais, foram realizados 400 sorteios aleatórios para cada tamanho de amostra, tendo-se obtido as respectivas estimativas de área de soja. No procedimento proposto, as amostras são distribuídas aleatoriamente em toda a área do estrato. Dessa maneira, a amostra irá respeitar a variabilidade espacial da cultura.

Neste procedimento, cada elemento da amostra teve sua localização geodésica definida sobre $\mathrm{o}$ mapa temático da soja. Por meio de uma consulta espacial, determinou-se a ocupação do solo (soja ou não-soja) para cada elemento que compôs a amostra. Essa informação foi utilizada na estimativa da área a partir do modelo de expansão direta, apresentada na equação (Thompson, 2002):

$$
\hat{\mathrm{Z}}_{\mathrm{c}}=\sum_{\mathrm{i}=1}^{\mathrm{m}} \mathrm{e}_{\mathrm{i}} \sum_{\mathrm{k}=1}^{\mathrm{n}_{\mathrm{i}}} \mathrm{Z}_{\mathrm{i}, \mathrm{k}}
$$

em que: $\hat{Z}_{c}$ é o estimador da área; $\mathrm{k}$ é o índice para representar o elemento amostrado com $\mathrm{k}=1, \ldots, \mathrm{n}_{\mathrm{i}}$; sendo $\mathrm{n}_{\mathrm{i}}$ o número de elementos amostrados no i-ésimo estrato; $\mathrm{e}_{\mathrm{i}}$ é o fator de expansão ou o inverso da probabilidade que um elemento amostral tem de estar na amostra escolhida no i-ésimo estrato, definido por $\mathrm{e}_{\mathrm{i}}\left(\mathrm{n}_{\mathrm{i}} / \mathrm{N}_{\mathrm{i}}\right)^{-1}$, em que $\mathrm{N}_{\mathrm{i}}$ é o número total de elementos no i-ésimo estrato, e $\mathrm{Z}_{\mathrm{i}, \mathrm{k}}$ é a área da cultura no k-ésimo elemento do i-ésimo estrato. $\mathrm{O} \mathrm{N}_{\mathrm{i}}$ do estrato foi calculado com base na área representada por cada elemento amostral, que foi de $25 \times 25 \mathrm{~m}\left(625 \mathrm{~m}^{2}\right)$, corresponde à resolução espacial do mapa temático de referência.

A variância foi estimada pela equação (Thompson, 2002):

$$
\mathrm{v}(\hat{\mathrm{Z}})=\sum_{\mathrm{i}=1}^{\mathrm{m}} \mathrm{N}_{\mathrm{i}}\left(\mathrm{N}_{\mathrm{i}}-\mathrm{n}_{\mathrm{i}}\right) \mathrm{s}_{\mathrm{i}}^{2} / \mathrm{n}_{\mathrm{i}}
$$

Pesq. agropec. bras., Brasília, v.45, n.6, p.585-592, jun. 2010 
em que $\mathrm{s}_{\mathrm{i}}{ }^{2}$ é a variância estimada da cultura no estrato i, definida por:

$$
\mathrm{s}_{\mathrm{i}}^{2}=\left[\sum_{\mathrm{k}=1}^{\mathrm{ni}}\left(\mathrm{z}_{\mathrm{ik}}-\overline{\mathrm{z}}_{\mathrm{i}}\right)^{2}\right] /(\mathrm{n}-1),
$$

em que $\bar{Z}_{i}$ é a área média da cultura no i-ésimo estrato, definida por:

$$
\overline{\mathrm{Z}}_{\mathrm{i}}=\sum_{\mathrm{k}=1}^{\mathrm{ni}} \mathrm{Z}_{\mathrm{ik}} / \mathrm{n}_{\mathrm{i}} .
$$

Um indicador disponível para avaliar a confiabilidade da estimativa de área via amostragem probabilística é o coeficiente de variação (CV), que é uma medida de dispersão padronizada que relaciona a média à variância e apresenta os resultados sob a forma de índice, o que permite comparações entre os valores de área plantada estimados pelos diferentes conjuntos amostrais. O CV (\%) é calculado pela equação (Thompson, 2002):

$$
\mathrm{CV}=\left[\mathrm{v}(\hat{\mathrm{Z}})^{0,5} / \hat{\mathrm{Z}}_{\mathrm{c}}\right] 100
$$

Para verificar a influência de dados discrepantes nos resultados, aplicou-se o método de reamostragem denominado bootstrap (Efron, 1982), com 1.000 repetições. O bootstrap é um método intensivo de análise estatística aplicável a modelos paramétricos e não paramétricos. Esse método consiste na obtenção de numerosas amostras, com reposição, a partir de uma amostra (Willmott et al., 1985). Cada nova amostra possui a probabilidade de $1 / \mathrm{n}$ de ser obtida. As amostras são escolhidas de forma aleatória e as estimativas recalculadas. Então, simula-se a curva função densidade de probabilidade da variável e estimam-se os valores de média, variância, mediana, assimetria e curtose, que permitem estabelecer o intervalo de confiança para as estimativas do modelo. Neste trabalho, utilizou-se o bootstrap para reduzir a influência de valores discrepantes, bem como para fornecer os parâmetros média, mínimo e máximo, que permitem a intercomparação dos resultados obtidos em cada conjunto amostral.

A exatidão das estimativas foi determinada por meio da diferença relativa (\%) em relação ao mapa temático de referência, pelo uso da equação:

$$
\text { Diferença relativa }=100\left(\hat{Z}_{c}-Z_{m}\right) / Z_{m} \text {, }
$$

em que: $Z_{m}$ é a área fornecida pelo mapa de referência e $\hat{Z}_{c}$ é o estimador de área.

A estratificação dos municípios, a alocação das amostras e os demais processamentos geográficos foram efetuados no Sistema de Processamento de Informações Georreferenciadas (SPRING) (Câmara et al., 1996).

\section{Resultados e Discussão}

Nos seis conjuntos amostrais, definidos em função dos valores de $\mathrm{E}$ e $\alpha$, o número de pontos amostrados variou de 203 a 1.990 (Tabela 2). Observa-se um aumento considerável no tamanho amostral do estrato 1 para o estrato 3. Além disso, a distribuição dos elementos amostrais ocorre, principalmente, em função da quantidade relativa da área de soja no estrato. Por exemplo, o estrato 1 perfaz $54 \%$ da área de estudo, mas concentra um número reduzido de elementos amostrais por possuir pouca área $(6,2 \%)$ ocupada com soja (Tabela 1$)$.

Em contraponto, o estrato 3 abrange apenas $25 \%$ da área em questão, mas envolve um número substancialmente maior de elementos amostrais por possuir cerca de $50 \%$ da área ocupada com a cultura da soja. Dessa forma, a estratificação assegura que os locais para a coleta de informação sobre a ocupação do solo concentrem-se nos municípios com maior área de soja, o que reduz o custo da visita às amostras em campo e aumenta a exatidão da estimativa. Esse resultado pode ser diferente em culturas como milho e café, em que a área cultivada apresenta grandes variações, conforme demonstrado por Adami et al. (2004).

A área cultivada com soja, presente no mapa temático de referência, foi de $24.929 \mathrm{~km}^{2}$ (Rizzi \& Rudorff, 2005). Observa-se que, com o aumento

\begin{tabular}{|c|c|c|c|c|c|c|}
\hline \multirow[t]{2}{*}{ Estrato } & \multicolumn{3}{|c|}{ Erro de 5,0} & \multicolumn{3}{|c|}{ Erro de 2,5} \\
\hline & A, $\alpha=10$ & $B, \alpha=5$ & $\mathrm{C}, \alpha=1$ & $\mathrm{D}, \alpha=10$ & $E, \alpha=5$ & $\mathrm{~F}, \alpha=1$ \\
\hline Estrato 1 & 44 & 63 & 109 & 177 & 251 & 434 \\
\hline Estrato 2 & 49 & 70 & 121 & 196 & 279 & 482 \\
\hline Estrato 3 & 110 & 155 & 269 & 438 & 622 & 1.074 \\
\hline Total & 203 & 288 & 499 & 811 & 1.152 & 1.990 \\
\hline
\end{tabular}
do tamanho amostral, a média das áreas estimadas

Tabela 2. Número de amostras por estrato para os seis conjuntos amostrais (A, B, C, D, E e F), definidos com base nos valores do erro de amostragem de 5,0 e $2,5 \%$ e nos níveis de significância $(\alpha)$ de 10,5 e $1 \%$.

Pesq. agropec. bras., Brasília, v.45, n.6, p.585-592, jun. 2010 
tende a aproximar-se do valor de referência (Tabela 3). Para o tamanho amostral F, com 1.990 pontos amostrados, o valor de área estimado pelo método bootstrap é de $24.895 \mathrm{~km}^{2}$, com o valor da diferença relativa de apenas $-0,14 \%\left(34 \mathrm{~km}^{2}\right)$. Pode-se observar que o tamanho amostral tem influência direta na precisão das estimativas. $\mathrm{O}$ maior e o menor $\mathrm{CV}$ médio foi observado para os conjuntos amostrais A $(22,32 \%)$ e F $(6,98 \%)$, respectivamente. Valores encontrados para os tamanhos de amostras E e F são similares aos reportados por diversos autores (Tsiligirides, 1998; Epiphanio et al., 2002; Adami et al., 2004, 2005, 2007; Rizzi et al., 2006) e foram classificados por Gomes (1984) como baixos. Os valores do CV para demais tamanhos de amostras foram classificados como médios (B, C e D) e altos (A). Assim, pode-se associar a significância dos indicadores estatísticos à significância prática, pois quanto menor o $\mathrm{CV}$, maior a probabilidade de as estimativas retornarem resultados condizentes com a realidade.

O conjunto amostral E (1.152 pontos) tem aproximadamente $60 \%$ do tamanho do conjunto amostral $\mathrm{F}$ (1.990 pontos). Apesar disso, os resultados obtidos por esse conjunto são satisfatórios, pois apresentam, em média, diferença relativa em torno de $2,6 \%$ e CV entre 7,5 e $11,1 \%$. Em $62 \%$ dos sorteios realizados, as estimativas ficaram dentro do intervalo entre -5 e $5 \%$ de diferença relativa. Assim, esse conjunto amostral possivelmente teria um custo menor para a obtenção da estimativa da área cultivada com soja, com incremento de $2,4 \%$ no CV em média (Tabela 3).

Como a área de estudo é uma região agrícola bem consolidada, pode-se considerar que existe boa estabilidade nas áreas destinadas às lavouras de produção de soja. Dessa maneira, o CV associado a cada tamanho amostral permanece estável ao longo dos anos, mesmo que ocorram variações conjunturais que influenciem na variação da área cultivada. Essa análise pode ser obtida a partir dos resultados do bootstrap que relata as amplitudes de $\mathrm{CV}$ e as diferenças relativas passíveis de ocorrerem em cada tamanho amostral (Tabela 3 ).

Observa-se que, com o aumento do tamanho amostral, a percentagem de estimativas dentro do intervalo de variação de 5\%, em módulo, também aumenta. Por exemplo, para o conjunto amostral F, 80,29\% das estimativasocorreram dentrodesseintervalodevariação. Além disso, para o tamanho de amostra, a média das estimativas de área das 400 repetições condiz com a área obtida pelo mapa temático de referência, o que indica a não existência de viés (Tabela 3 ). Isso reforça a assertiva de que o aumento do tamanho amostral reduz o $\mathrm{CV}$ e, consequentemente, fornece estimativas mais próximas àrealidade. Nos demais tamanhos deamostras, os valores máximos, mínimos e, principalmente, a média das diferenças relativas indicam tendência de superestimar a área plantada com soja. As maiores frequências de diferenças relativas positivas foram observadas para as amostras com menor número (tamanho) de pontos sorteados (Figura 2).

Diante disso, acredita-se que o número de pontos, ou unidades amostrais, adequado para a estimativa de área de soja na região estudada está em torno de 1.990. Cabe ressaltar que, mesmo para o estrato que concentra o maior número de pontos amostrais (estrato 3, 1.074 pontos e 71 municípios), cada município terá, em média, apenas 15 pontos que podem ser localizados no campo de maneira relativamente fácil com o auxílio de um receptor GPS.

O trabalho de campo também pode ser minimizado com a eventual utilização de imagens

Tabela 3. Valores mínimos, máximos e médios de área de soja, diferença relativa e coeficiente de variação (CV), estimados pelo método bootstrap, e percentual de estimativas dentro do intervalo de variação (IV) de $5 \%$ de diferença relativa, em cada conjunto amostral.

\begin{tabular}{|c|c|c|c|c|c|c|c|c|c|c|c|}
\hline \multirow{2}{*}{$\begin{array}{l}\text { Conjunto } \\
\text { amostral }\end{array}$} & \multirow{2}{*}{$\begin{array}{c}\text { Número } \\
\text { de pontos }^{(1)}\end{array}$} & \multicolumn{3}{|c|}{ Área de soja $\left(\mathrm{km}^{2}\right)$} & \multicolumn{3}{|c|}{ Diferença relativa $(\%)$} & \multicolumn{3}{|c|}{$\mathrm{CV}(\%)$} & \multirow[t]{2}{*}{ IV $(\%)$} \\
\hline & & Mínimo & Máximo & Média & Mínimo & Máximo & Média & Mínimo & Máximo & Média & \\
\hline A & 203 & 19.884 & 37.651 & 27.964 & $-20,2$ & 51,0 & 12,2 & 10,2 & 31,9 & 22,3 & 20,2 \\
\hline B & 288 & 19.849 & 36.506 & 27.621 & $-20,4$ & 46,4 & 10,8 & 9,8 & 25,9 & 19,0 & 19,8 \\
\hline $\mathrm{C}$ & 499 & 20.688 & 33.042 & 26.837 & $-17,0$ & 32,5 & 7,6 & 9,2 & 18,8 & 14,5 & 33,0 \\
\hline $\mathrm{D}$ & 811 & 21.796 & 30.686 & 26.127 & $-12,6$ & 23,1 & 4,8 & 8,8 & 14,3 & 11,3 & 49,4 \\
\hline E & 1.152 & 21.650 & 29.315 & 25.575 & $-13,2$ & 17,6 & 2,6 & 7,5 & 11,1 & 9,4 & 62,1 \\
\hline $\mathrm{F}$ & 1.990 & 22.123 & 27.652 & 24.895 & $-11,3$ & 10,9 & $-0,1$ & 5,9 & 8,1 & 7,0 & 80,3 \\
\hline
\end{tabular}

${ }^{(1)}$ Número total de elementos amostrais (pontos, $n$ ) selecionados para estimar a área com um determinado nível de significância e um determinado erro de amostragem. 
de sensoriamento remoto atuais, conforme Figura 3, que apresenta alguns exemplos típicos do uso de imagens de satélite de sensoriamento remoto para este fim. Na Figura 3 A, identificam-se inicialmente dois pontos que estão localizados em um corpo d'água (pontos 129 e 390). A Figura 3 B apresenta o ponto 765 que, por estar sob nuvens, obriga o trabalho de campo para a identificação do uso do solo. Os pontos 246 (Figura 3 C) e 570 (Figura 3 A) representam áreas com e sem soja, respectivamente. A identificação desses pontos como área de soja ou como área sem soja segue a caracterização espectral apresentada por Rizzi \& Rudorff (2005) para imagens TM/Landsat-5 e ETM+/Landsat-7.

Desse modo, as imagens de satélites de sensoriamento remoto podem fornecer informações a respeito de pontos amostrais selecionados sobre áreas de fácil identificação visual e que claramente não são de soja (água, área urbana, floresta, entre outros) (Moreira, 1991; Rizzi \& Rudorff, 2005). Este trabalho de exclusão das áreas que não sejam de soja pode ser realizado com auxílio das imagens recentes disponíveis no Google Earth, uma vez que tais usos e ocupação do solo normalmente não são rapidamente convertidos para área de cultivo de soja.

Os pontos selecionados para compor a amostra de área podem ser visitados no campo por ocasião da época de implantação da cultura. Como a estimativa de área pode ser obtida tão logo o levantamento da informação sobre os pontos sorteados esteja finalizado (Adami et al., 2004), a estimativa da área de soja pode ser realizada em tempo hábil e estar
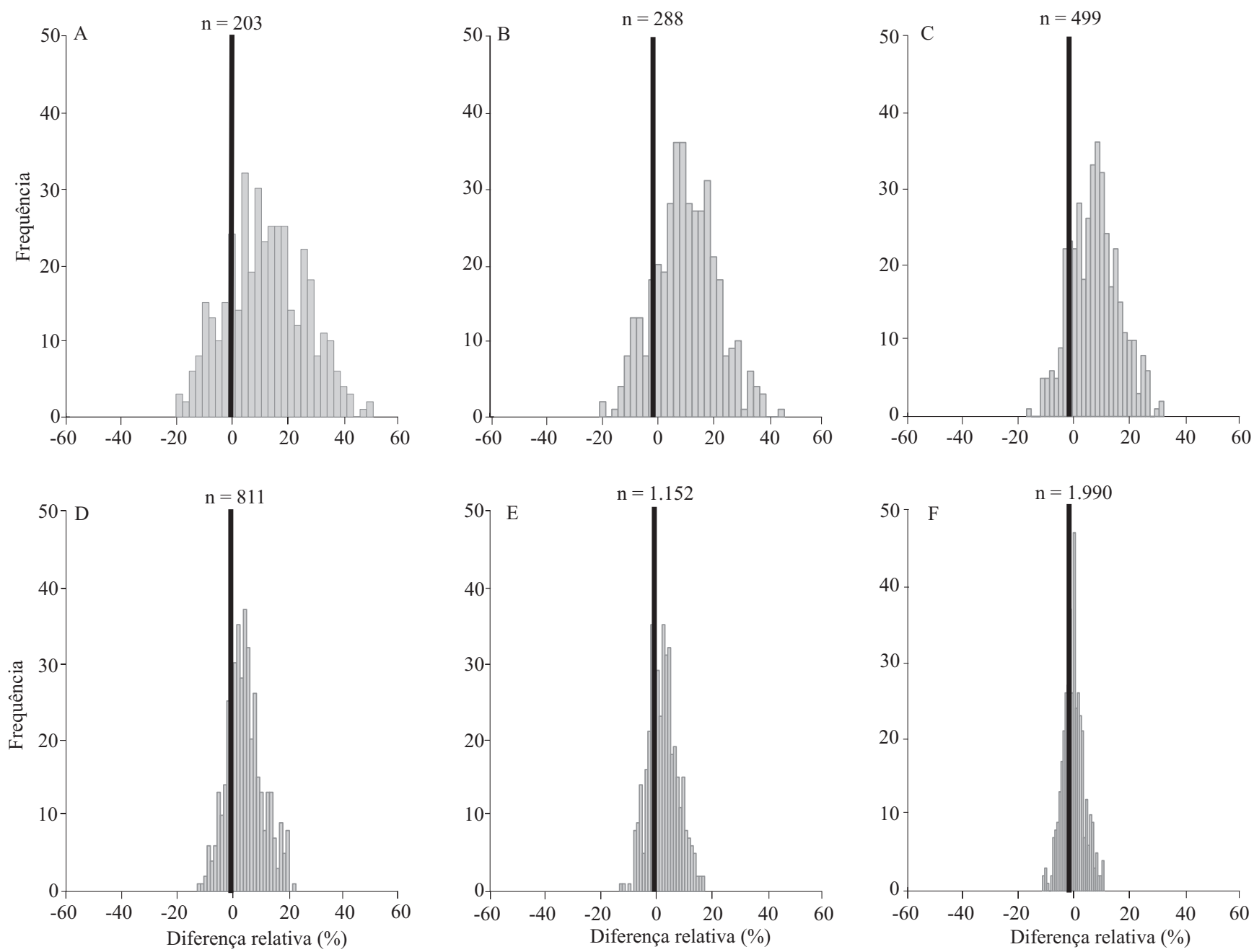

Figura 2. Frequência de ocorrência de diferenças relativas (\%) entre as estimativas oriundas do sistema de amostragem e aquela proveniente do mapa de referência, para os conjuntos amostrais A, B, C, D, E e F. 


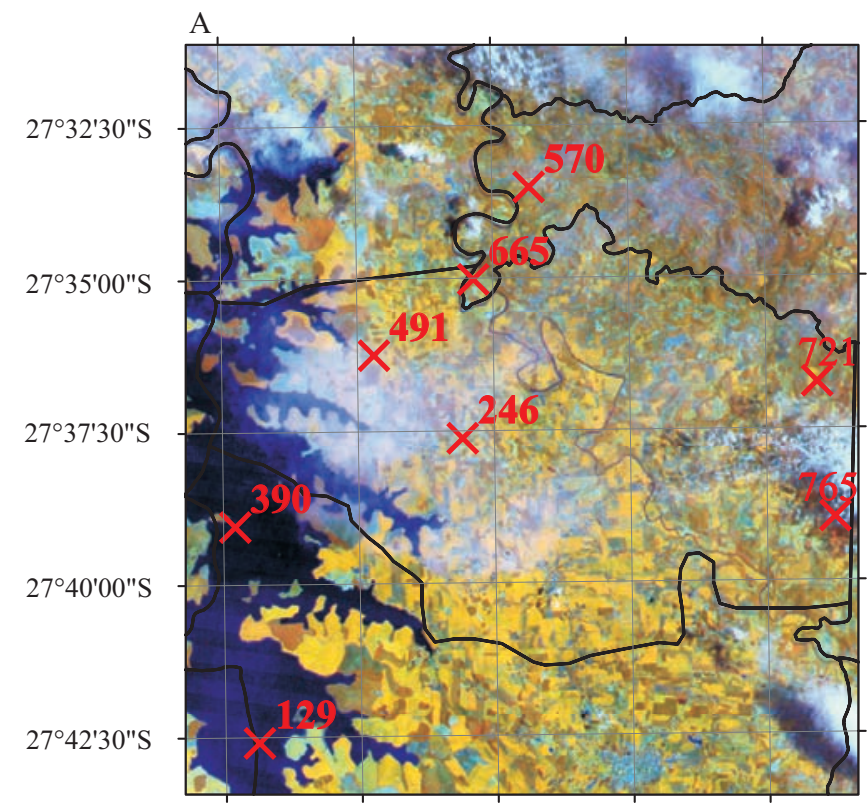

$52^{\circ} 45^{\prime} 00^{\prime \prime} \mathrm{W} 52^{\circ} 42^{\prime} 30^{\prime \prime} \mathrm{W} 52^{\circ} 40^{\prime} 00^{\prime \prime} \mathrm{W} 52^{\circ} 37^{\prime} 30^{\prime \prime} \mathrm{W} 52^{\circ} 35^{\prime} 00^{\prime \prime} \mathrm{W}$

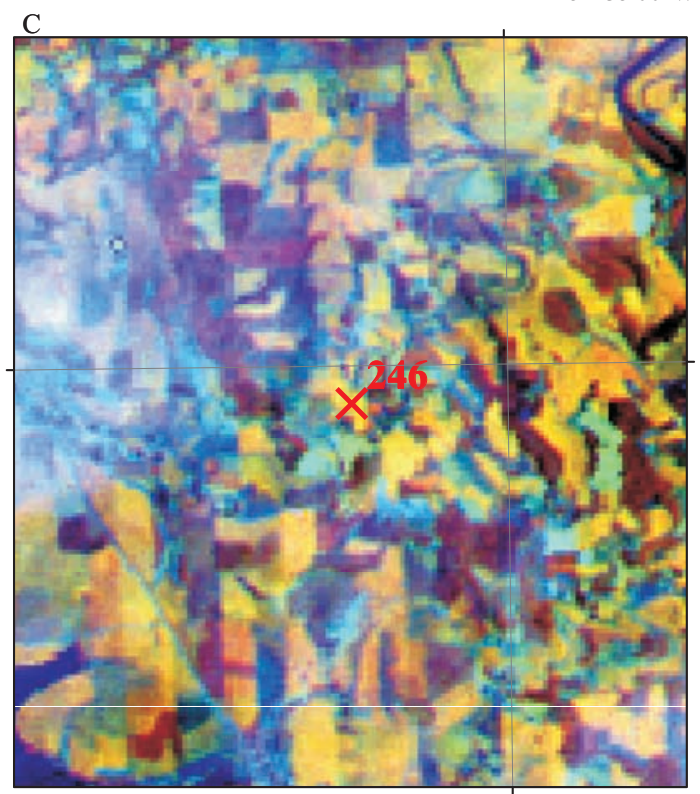

$52^{\circ} 40^{\prime} 00^{\prime \prime} \mathrm{W}$

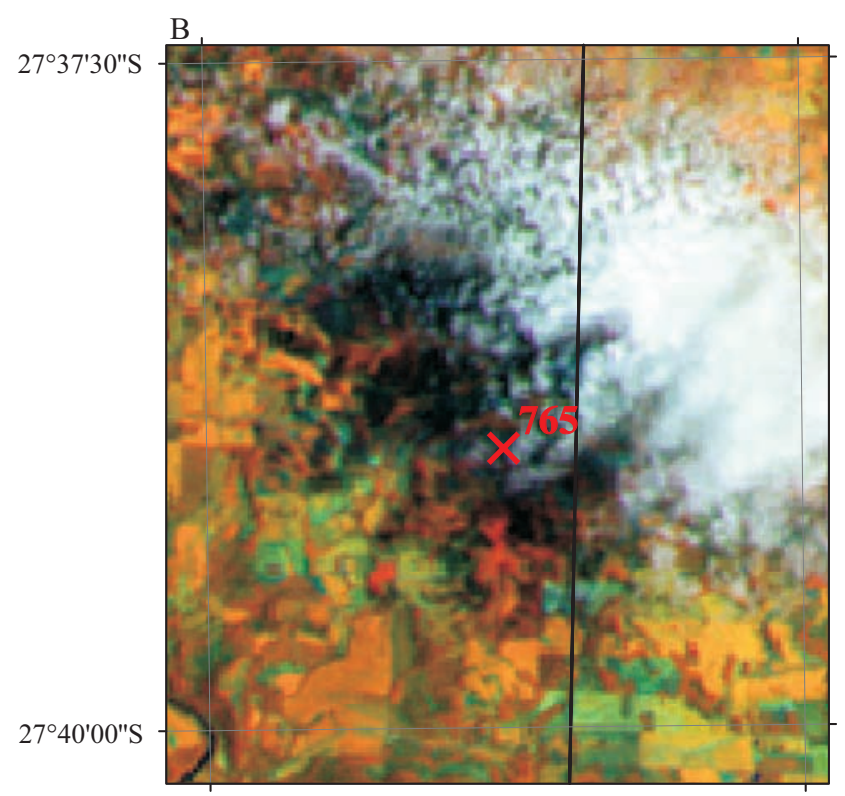

$52^{\circ} 35^{\prime} 00^{\prime \prime} \mathrm{W}$

$52^{\circ} 32^{\prime} 00^{\prime \prime} \mathrm{W}$

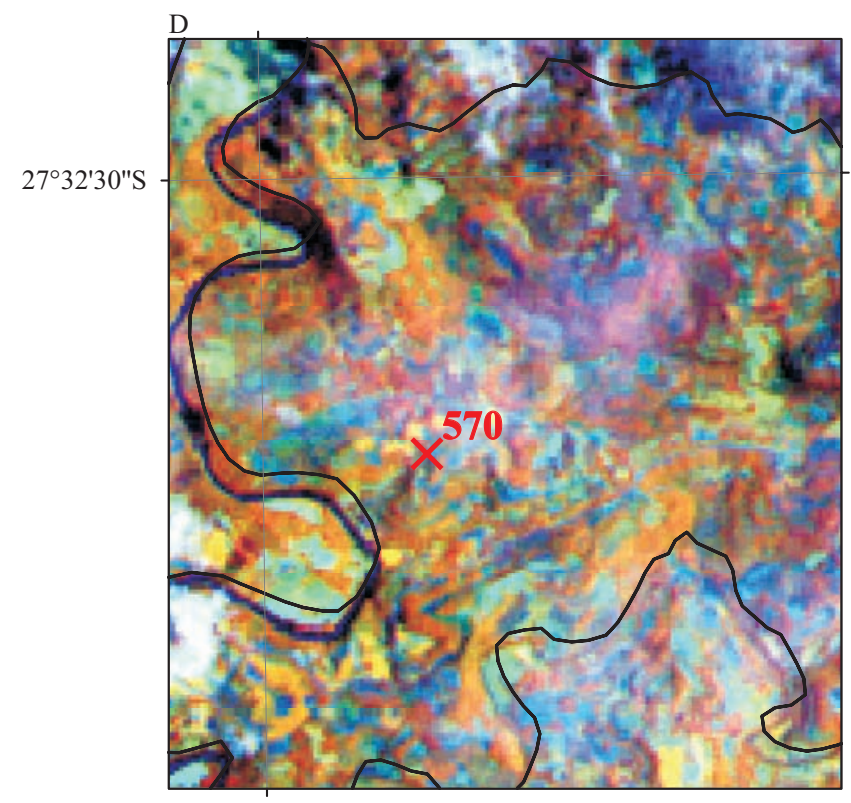

$52^{\circ} 40^{\prime} 00^{\prime \prime} \mathrm{W}$

Figura 3. Exemplo do uso da imagem de satélite TM/Landsat-5 (órbita 222 ponto 79, adquirida em 8/1/2001) para reduzir o número de pontos visitados a campo, em que: $\mathrm{A}$, visão sinótica sobre alguns pontos sorteados; $\mathrm{B}$, ponto sob nuvem com necessidade de ser identificado a campo; C, ponto com soja; e D, ponto sem soja.

associada a bons indicadores estatísticos. Com exceção da estratificação e do tamanho amostral, que devem ser adaptados às características regionais e a distribuição espacial da cultura, a metodologia apresentada pode ser utilizada para estimar a área plantada de qualquer cultura.

\section{Conclusão}

É possível estimar a área cultivada de soja no Rio Grande do Sul por meio de um modelo de amostragem aleatória estratificada por pontos, com no mínimo 1.152 pontos de amostra, de modo a fornecer informações objetivas e em tempo oportuno. 


\section{Referências}

ADAMI, M.; MOREIRA, M.A.; RUDORFF, B.F.T.; FREITAS, C. da C. Análise da eficiência dos estimadores de expansão direta e de regressão par a áreas cultivadas com café, milho e soja no município de Cornélio Procópio, estado do Paraná. Agricultura em São Paulo, v.51, p.5-13, 2004.

ADAMI, M.; MOREIRA, M.A.; RUDORFF, B.F.T.; FREITAS, C. da C.; FARIA, R.T de. Expansão direta na estimativa de culturas agrícolas por meio de segmentos regulares. Revista Brasileira de Cartografia, v.1, p.22-27, 2005.

ADAMI, M.; MOREIRA, M.A.; RUDORFF, B.F.T.; FREITAS, C. da C.; FARIA, R.T. de; DEPPE, F.A.C. Painel amostral para estimativa de áreas agrícolas. Pesquisa Agropecuária Brasileira, v.42, p.81-88, 2007.

CÂMARA, G.; SOUZA, R.C.M.; FREITAS, U.M.; GARRIDO, J. SPRING: integrating remote sensing and GIS by object-oriented data modelling. Computers and Graphics, v.20, p.395-403, 1996.

EFRON, B. The Jackknife, the Bootstrap, and other resampling plans. Philadelphia: Society for Industrial and Applied Mathematics, 1982. 92p.

EPIPHANIO, J.C.N.; LUIZ, A.J.B.; FORMAGGIO, A.R. Estimativa de áreas agrícolas municipais, utilizando sistema de amostragem simples sobre imagens de satélite. Bragantia, v.61, p.187-197, 2002.

FIGUEIREDO, D.C. Projeto GeoSafras: aperfeiçoamento do sistema de previsão de safras da Conab. Revista de Política Agrícola, v.14, p.110-120, 2005.

INSTITUTO BRASILEIRO DE GEOGRAFIA E ESTATÍSTICA. Produção agrícola municipal. Rio de Janeiro: IBGE, 2001. Disponível em: <http://www.sidra.ibge.gov.br/bda/tabela/listabl. asp? $\mathrm{z}=\mathrm{t} \& \mathrm{c}=1612>$. Acesso em: 15 maio 2009.

GOMES, F.P. A estatística moderna na pesquisa agropecuária. Piracicaba: Associação Brasileira para Pesquisa da Potassa e do Fosfato, 1984. 160p.

LUIZ, A.J.B. Estatísticas agrícolas por amostragem auxiliadas pelo sensoriamento remoto. 2003. 112p. Tese (Doutorado) Instituto Nacional de Pesquisas Espaciais, São José dos Campos.

LUIZ, A.J.B.; NEVES, M.C.; EVANGELISTA, S.R.M.; MAIA, A. de H.N. Sistema para estimação de área plantada por amostragem - SEARA - proposta de delineamento amostral para café em São Paulo e para grãos em Minas Gerais. Jaguariúna: Embrapa Meio Ambiente, 2006. 27p. (Embrapa Meio Ambiente. Documentos, 49).

LUIZ, A.J.B.; OLIVEIRA, J.C.; EPIPHANIO, J.C.N.; FORMAGGIO, A.R. Auxílio das imagens de satélite aos levantamentos por amostragem em agricultura. Agricultura em São Paulo, v.49, p.41-54, 2002.
MOREIRA, M.A. Expansão direta para estimar áreas de soja e milho através de dados multiespectrais e temporais do Landsat/TM. Pesquisa Agropecuária Brasileira, v.26, p.1607-1613, 1991.

MOREIRA, M.A.; BARROS, M.A.; FARIA, V.G.C.; ADAMI, M. Tecnologia de informação: imagens de satélite para o mapeamento de áreas de café de Minas Gerais. Informe Agropecuário, v.28, p.27-37, 2007.

MUELleR, C.C.; SILVA, G.; VILlAlOBOS, A.G. Pesquisa agropecuária do Paraná - safra 1986/87 (Programa de Aperfeiçoamento das Estatísticas Agropecuárias). Revista Brasileira de Estatística, v.49, p.55-84, 1988.

PINO, F.A. Estimação subjetiva de safras agrícolas. Informações Econômicas, v.31, p.55-58, 2001.

RIZZI, R.; RUDORFF, B.F.T. Estimativa da área de soja no Rio Grande do Sul por meio de imagens Landsat. Revista Brasileira de Cartografia, v.57, p.226-234, 2005.

RIZZI, R.; RUDORFF, B.F.T; ADAMI, M. Estimativa da área de soja no Estado do Rio Grande do Sul por um método de amostragem. Ciência Rural, v.36, p.30-35, 2006.

RUDORFF, B.F.T.; AGUIAR, D.A.; SILVA, W.F.; SUGAWARA, L.M.; ADAMI, M.; MOREIRA, M.A. Studies on the rapid expansion of sugarcane for ethanol production in São Paulo State (Brazil) using Landsat Data. Remote Sensing, v.2, p.1057-1076, 2010.

RUDORFF, B.F.T.; BERKA, L.M.S.; MOREIRA, M.A.; DUARTE, V.; XAVIER, A.C.; ROSA, V.G.C.; SHIMABUKURO, Y.E. Imagens de satélite no mapeamento e estimativa de área de cana-de-açúcar em São Paulo: ano safra 2003/04. Agricultura em São Paulo, v.52, p.21-39, 2005.

SANO, E.E.; FERREIRA, L.G.; ASNER, G.P.; STEINKE, E.T. Spatial and temporal probabilities of obtaining cloud-free Landsat images over the Brazilian tropical savanna. International Journal of Remote Sensing, v.28, p.2739-2752, 2007.

SUGAWARA, L.M.; RUDORFF, B.F.T.; ADAMI, M. Viabilidade de uso de imagens do Landsat em mapeamento de área cultivada com soja no Estado do Paraná. Pesquisa Agropecuária Brasileira, v.43, p.1763-1768, 2008.

THOMPSON, S.K. Sampling. 2.ed. New York: John Wiley \& Sons, 2002. 343p.

TSILIGIRIDES, T.A. Remote sensing as a tool for agricultural statistics: a case study of area frame sampling methodology in Hellas. Computers and Electronics in Agriculture, v.20, p.45-77, 1998.

WILLMOTT, C.J.; ACKLESON, S.G.; DAVIS, R.E.; FEDDEMA, J.J.; KLINK, K.M.; LEGATES, D.R.; O’DONNELL, J.; ROWE, C.M. Statistics for the evaluation and comparison of models. Journal of Geophysical Research, v.90, p.8995-9005, 1985.

Recebido em 7 de outubro de 2009 e aprovado em 10 de maio de 2010 